\title{
Hall effect on steady mhd flow and heat transfer of a viscous fluid in a rectangular channel with suction and injection
}

\author{
Ramana Murthy Josyula Venkata ${ }^{1, *}$, and Pavankumar Reddy Muduganti ${ }^{2}$ \\ ${ }^{1}$ Department of Mathematics, National Institute of Technology, Warangal-506004, Telangana, India \\ ${ }^{2}$ Department of Mathematics, National Institute of Technology, Warangal-506004, Telangana, India
}

\begin{abstract}
The flow of an incompressible viscous fluid under the influence of an applied uniform magnetic field in a rectangular channel with suction at the adjacent two side walls is studied by considering Hall current and Joule heating effects. The rectangular channel is subjected to a uniform suction from top wall and injection from right wall. An external uniform magnetic field is applied perpendicular to the flow. Two sides (left and bottom) of the channel are kept at two constant but different temperature and other two sides (right and top) are maintained at constant heat flux. Viscous and Joule dissipations are considered in the energy equation. The relevant equations of motion are solved numerically to yield the velocity and the temperature distribution. The current density is also studied.
\end{abstract}

\section{Introduction}

Berman $[1,2]$ has studied the effect of suction and injection in the absence of magnetic field on the laminar fluid flow through channels of various geometries. The steady flow of a conducting fluid through a straight avenue under a uniform transverse magnetic field presents one of the elementary problems in magneto hydrodynamics. Magnetic flow in a rectangular channel is a classical problem that has significant applications in magneto hydrodynamic power generators and pumps etc. Nowadays, magnetic field has earned great value due to widespread applications in industry and bioengineering, such as electrostatic precipitation, power generators, petroleum industry, aerodynamic heating, the purification of metals from non-metallic inclusions, polymer technology and fluid droplet sprays. Hartmann[3] was the first person to obtain a solution for this type of flows to compare with his experimental results on mercury. Hartmann and Lazarus [4] studied the impact of a transverse uniform magnetic field on the flow of a viscous incompressible electrically conducting fluid between two infinite parallel stagnant and insulating plates. Hunt [5], Mehta and Jain [6], Lundgren et al [7], Mandal [8], studied the laminar hydro-magnetic flow in a rectangular channel. Ramana Murthy and Bahali [9], Sai and Nageswara Rao [10] studied MHD flow in a rectangular channel with suction and injection imposed on opposite walls without considering the Hall effect. Soundalgekar et al [11], Soundalgekar and Uplekar [12] studied the MHD flow and heat transfer between two parallel plates with Hall effect. An approximate method of solution has given by Tani [13] for the steady laminar incompressible flow of an electrically conducting fluid with consideration of Hall effect through a rectangular channel of arbitrary cross section with conducting or non-conducting walls in the presence of a uniform transverse magnetic field based on a minimum principle. MHD flow and heat transfer in a rectangular channel with Hall effect was investigated by Ahmed and Attia [14, 15], Hassan and Attia [16], Srnivasacharya and Shiferaw [17].

In the above studies of non-Newtonian fluids with MHD effect, Hall effect with suction and injection in a rectangular channel has not been considered. Attia and Ahmed [18] and Attia [19] studied Hall effect on MHD flow and heat transfer between parallel plates with suction and injection of Bingham and dusty fluids respectively. As far as the authors know, Hall effect of the magneto hydrodynamic flow of viscous fluid through a rectangular channel with suction and injection at the adjacent walls has been paid less attention.

Hence, in this paper, our objective is to study the Hall effect of the magneto hydrodynamic flow of viscous fluid through a rectangular channel with suction/ injection at the adjacent walls. We have used Cartesian co-ordinate system for formulating the mathematical equations and obtained the numerical solution for stream function, temperature and current density. The effects of physical parameters like Reynolds number, Hartmann number, Hall parameter and suction parameter on stream lines, isothermal lines and current density are studied and illuminated the results through graphs.

\section{Mathematical Formulation}

The geometry of the problem is shown in Fig. 1. The two dimensional incompressible laminar viscous flow through a rectangular tube due to suction/injection at the neighboring walls is considered. The Cartesian coordinate system with origin at the bottom left corner

* Corresponding author: jvr@nitw.ac.in 
and $X$ and $Y$ axes along the walls is taken. The tube is of length $a$ and height $b$. Injection with velocity $V_{1}$ at the wall $X=a$ and Suction with velocity $V_{2}$ at the top wall $Y=b$ are imposed. The flow is driven by an applied uniform suction from right wall and injection from top wall. A uniform magnetic field with magnetic flux density vector $H$ is applied perpendicular to the flow which is assumed to be also the total magnetic field. The induced magnetic field is neglected by assuming a very small magnetic Reynolds number. The Hall effect is taken into consideration.

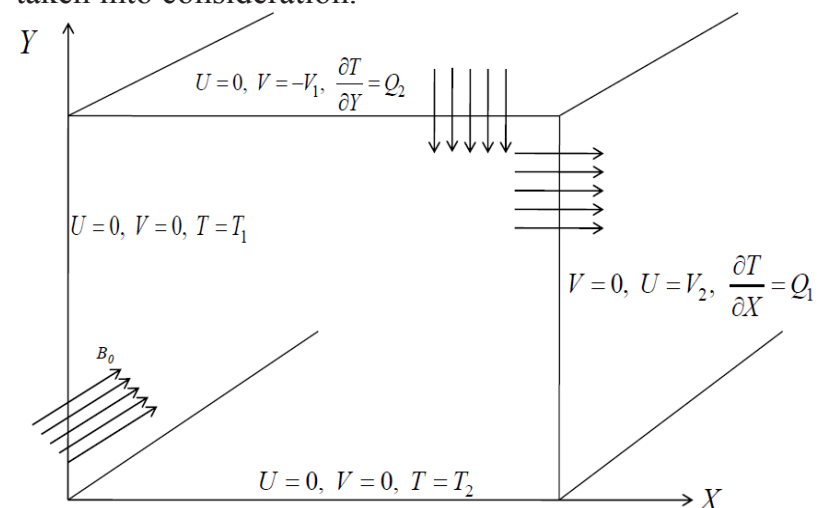

Fig.1: Flow configuration in a rectangular channel with suction

Velocity of fluid satisfies impermeability condition and no slip condition at the walls implies that $U=0, V=0$ at $X=0$ and $U=0, V=0$ at $Y=0$. Suction and no slip condition at the permeable walls implies that $U=V_{2}, V=0$ at $X=a$ and $U=0, V=-V_{1}$ at $Y=b$. The temperature field satisfies constant temperature on the impermeable walls implies that $T=T_{1}$ at $X=0$ and $T=T_{2}$ at $Y=0$ and constant heat flux at the permeable walls implies that $\partial T / \partial X=Q_{1}$ at $X=a$ and $\partial T / \partial Y=Q_{2}$ at $Y=b$. Thus, the velocity vector of the fluid is given by

$$
Q=U \dot{i}+V j
$$

It is because of the conservation of mass,

$$
\text { i.e., } \nabla \cdot \boldsymbol{Q}=0
$$

If the Hall term is retained, the current density $\boldsymbol{I}^{*}$ is given by

$$
\boldsymbol{I}^{*}=\sigma\left\{\boldsymbol{Q} \wedge \boldsymbol{H}-\beta\left(\boldsymbol{J}^{*} \wedge \boldsymbol{H}\right)\right\}
$$

where $\sigma$ is the electric conductivity of the fluid, $\beta$ is the Hall factor and $\boldsymbol{H}=B_{0} \boldsymbol{k}, B_{0}$ is constant. Eq. (2) may be solved in $\boldsymbol{I}^{*}$ to yield the Lorentz force vector in the form

$$
\boldsymbol{I}^{*} \wedge \boldsymbol{H}=\frac{-\sigma B_{0}^{2}}{1+m^{2}}\{(U-m V) \boldsymbol{i}+(V+m U) \boldsymbol{j}\}
$$

where $m=\sigma \beta B_{0}$ is the Hall parameter. The fluid motion is governed by the momentum equations

$$
\begin{aligned}
& \rho\left(U \frac{\partial U}{\partial X}+V \frac{\partial U}{\partial Y}\right)=-\frac{\partial P}{\partial X}+\mu\left(\frac{\partial^{2} U}{\partial X^{2}}+\frac{\partial^{2} U}{\partial Y^{2}}\right)-\frac{\sigma B_{0}^{2}}{1+m^{2}}(U-m V) \\
& \rho\left(U \frac{\partial V}{\partial X}+V \frac{\partial V}{\partial Y}\right)=-\frac{\partial P}{\partial Y}+\mu\left(\frac{\partial^{2} V}{\partial X^{2}}+\frac{\partial^{2} V}{\partial Y^{2}}\right)-\frac{\sigma B_{0}^{2}}{1+m^{2}}(V+m U)
\end{aligned}
$$

where $\rho$ is the density of the fluid, $P$ is pressure and $\mu$ is the coefficient of viscosity.

The temperature distribution is governed by the energy equation

$$
\rho c_{p} \frac{d T}{d t}=k \nabla^{2} T+\frac{\sigma B_{0}^{2}}{1+m^{2}}\left(U^{2}+V^{2}\right)+\mu\left(\left(\frac{\partial U}{\partial X}\right)^{2}+\left(\frac{\partial V}{\partial Y}\right)^{2}\right)
$$

where $c_{p}$ is specific heat capacity and $k$ is the thermal conductivity of the fluid. The second and third terms on the right-hand side represent the Joule and viscous dissipations respectively. Eqs. (4)-(6) can be made dimensionless by introducing the following dimensionless variables:

$$
\begin{gathered}
X=a x, Y=a y, U=V_{1} u, V=V_{1} v, P=\rho V_{1}^{2} p, \\
t=\frac{a}{V_{1} \tau}, T=T_{1}+\theta\left(T_{2}-T_{1}\right)
\end{gathered}
$$

We also define the following dimensionless parameters: $V_{0}=\frac{V_{2}}{V_{1}}$, the suction parameter,

$R e=\frac{\rho V_{1} a}{\mu}$, the Reynolds number,

$H a=B_{0} a \sqrt{\sigma / \mu}$, the Hartmann number,

$P e=R e * \operatorname{Pr}=\frac{\rho c_{p} V_{1} a}{k}$, the Peclet number,

$\operatorname{Pr}=\frac{\mu c_{p}}{k}$, the Prandtl number,

$E_{c}=\frac{V_{1}^{2}}{c_{p}\left(T_{2}-T_{1}\right)}$, the Eckert number.

In terms of these dimensionless quantities, Eqs. (4)-(6) may be written as

$$
\begin{aligned}
& u \frac{\partial u}{\partial x}+v \frac{\partial u}{\partial y}=-\frac{\partial p}{\partial x}+\frac{1}{R e} \nabla^{2} u-\frac{H a^{2}}{R e\left(1+m^{2}\right)}(u-m v) \\
& u \frac{\partial v}{\partial x}+v \frac{\partial v}{\partial y}=-\frac{\partial p}{\partial y}+\frac{1}{R e} \nabla^{2} v-\frac{H a^{2}}{R e\left(1+m^{2}\right)}(v+m u) \\
& u \frac{\partial \theta}{\partial x}+v \frac{\partial \theta}{\partial y}=\frac{1}{P e} \nabla^{2} \theta+\frac{H a^{2} E c}{\operatorname{Re}\left(1+m^{2}\right)}\left(u^{2}+v^{2}\right)+\frac{E c}{R e}\left[\left(\frac{\partial u}{\partial x}\right)^{2}+\left(\frac{\partial v}{\partial y}\right)^{2}\right]
\end{aligned}
$$

\section{Solution of the problem}

\subsection{Stream function}

We introduce stream function $\psi$ as below such that equation (1) is satisfied.

$$
u=\frac{\partial \psi}{\partial y} \text { and } v=-\frac{\partial \psi}{\partial x} .
$$

Eliminating pressure term from (7)-(8) and substituting (10), we get the equation for the stream function as

$$
\frac{\partial \psi}{\partial y} \frac{\partial}{\partial x}\left(\nabla^{2} \psi\right)-\frac{\partial \psi}{\partial x} \frac{\partial}{\partial y}\left(\nabla^{2} \psi\right)=\frac{1}{R e} \nabla^{4} \psi-\frac{H a^{2}}{R e\left(1+m^{2}\right)} \nabla^{2} \psi .
$$

The initial and boundary conditions for the velocity in the dimensionless form are written as

$$
\begin{gathered}
\frac{\partial \psi}{\partial x}=0 \text { on } x=0 \text { and on } x=1 \\
\frac{\partial \psi}{\partial y}=0 \text { on } y=0 \text { and on } y=y_{0} \\
\frac{\partial \psi}{\partial y}=0 \text { on } x=0 \text { and } \frac{\partial \psi}{\partial y}=V_{0} \text { on } x=1 \\
\frac{\partial \psi}{\partial x}=0 \text { on } y=0 \text { and } \frac{\partial \psi}{\partial x}=1 \text { on } y=y_{0}
\end{gathered}
$$

These conditions, by integrating, are converted in to the conditions on $\psi$ as follows:

$$
\left.\begin{array}{c}
\frac{\partial \psi}{\partial x}=0 \text { on } x=0 \text { and on } x=1 \\
\psi=0 \text { on } x=0 \text { and on } y=0 \\
\psi=x \text { on } y=y_{0} \text { and } \psi=V_{0} y \text { on } x=1 \\
\frac{\partial \psi}{\partial y}=0 \text { on } y=0 \text { and on } y=y_{0}
\end{array}\right\}
$$

We solve the equation (11) with conditions (12) by Finite Difference Method. The cavity is covered with a 
mesh of step size $h$ with $(M-1)$ intervals on $X$ direction and $(N-1)$ intervals on $Y$ direction. For each grid point $(i$, $j$ ) within the cavity, the $4^{\text {th }}$ order term is approximated by 13 point scheme as given in Titus Petrila and Damian Trif [20] and Pozrikidis [21] and harmonic terms by $2^{\text {nd }}$ order 5 point scheme. Then equation (11) can be written as below:

$$
\begin{aligned}
& 80 \psi_{i, j}^{(n)}-32\left(\psi_{i+1, j}^{(n)}+\psi_{i-1, j}^{(n)}+\psi_{i, j+1}^{(n)}+\psi_{i, j-1}^{(n)}\right) \\
& +8\left(\psi_{i+1, j+1}^{(n)}+\psi_{i-1, j+1}^{(n)}+\psi_{i+1, j-1}^{(n)}+\psi_{i-1, j-1}^{(n)}\right) \\
& +4\left(\psi_{i, j+2}^{(n)}+\psi_{i, j-2}^{(n)}+\psi_{i+2, j}^{(n)}+\psi_{i-2, j}^{(n)}\right) \\
& -\frac{4 h^{2} \cdot H a^{2}}{\left(1+m^{2}\right)}\left(\psi_{i+1, j}^{(n)}+\psi_{i-1, j}^{(n)}-4 \psi_{i, j}^{(n)}+\psi_{i, j+1}^{(n)}+\psi_{i, j-1}^{(n)}\right) \\
& +\operatorname{Re}\left[\begin{array}{c}
\left(\psi_{i+1, j}^{(n-1)}-\psi_{i-1, j}^{(n-1)}\right)\left(\begin{array}{c}
\psi_{i+1, j+1}^{(n)}-\psi_{i+1, j-1}^{(n)} \\
+\psi_{i-1, j+1}^{(n)}-\psi_{i-1, j-1}^{(n)}-4 \psi_{i, j+1}^{(n)} \\
+4 \psi_{i, j-1}^{(n)}+\psi_{i, j+2}^{(n)}-\psi_{i, j-2}^{(n)}
\end{array}\right) \\
-\left(\psi_{i, j+1}^{(n-1)}-\psi_{i, j-1}^{(n-1)}\right)\left(\begin{array}{c}
\psi_{i+2, j}^{(n)}-\psi_{i-2, j}^{(n)} \\
-4 \psi_{i+1, j}^{(n)}+4 \psi_{i-1, j}^{(n)}+\psi_{i+1, j+1}^{(n)} \\
-\psi_{i-1, j+1}^{(n)}+\psi_{i+1, j-1}^{(n)}-\psi_{i-1, j-1}^{(n)}
\end{array}\right)
\end{array}\right)=0
\end{aligned}
$$

for $i=2,3, \ldots, M-1$ and $j=2,3, \ldots, N-1$.

For the derivative boundary conditions, we used central difference scheme. The nodes numbering is as follows:

\begin{tabular}{|l|l|}
\hline Along $X$ direction & Along $Y$ direction \\
\hline $\begin{array}{l}x_{1} \text { node on the boundary } \\
x=0 .\end{array}$ & $\begin{array}{l}y_{1} \text { node on the boundary } \\
y=0 .\end{array}$ \\
\hline $\begin{array}{l}x_{2}, x_{3}, \ldots, x_{M-1} \text { inside the } \\
\text { computational domain }\end{array}$ & $\begin{array}{l}y_{2}, y_{3}, \ldots, y_{N-1} \text { inside the } \\
\text { computational domain }\end{array}$ \\
\hline $\begin{array}{l}x_{M} \text { node on the boundary } \\
x=1\end{array}$ & $\begin{array}{l}y_{N} \text { node on the boundary } \\
y=y_{0}\end{array}$ \\
\hline
\end{tabular}

Thus at $x=0$ (i.e., $i=1$ ) we will

have, $\psi(0, y)=0$ and $\frac{\partial \psi}{\partial x}=0$. These conditions are

taken as

$$
\begin{aligned}
& \psi_{1, j}=0 \text { and } \frac{\psi_{2, j}-\psi_{0, j}}{2 h}=0 \\
& \text { or } \psi_{2, j}=\psi_{0, j} \text { for } j=1,2, \ldots, N
\end{aligned}
$$

at $x=1$ ( i.e., $i=M$ ) we will have,

$\psi(1, y)=V_{0} y$ and $\frac{\partial \psi}{\partial x}=0$. These conditions are taken

as

$$
\begin{aligned}
& \psi_{M, j}=V_{0}(j-1) h \text { and } \frac{\psi_{M+1, j}-\psi_{M-1, j}}{2 h}=0 \\
& \text { or } \psi_{M+1, j}=\psi_{M-1, j} \text { for } j=1,2, \ldots, N
\end{aligned}
$$

Similarly at $y=0$ we have,

$$
\psi_{i, 1}=0 \text { and } \psi_{i, 2}=\psi_{i, 0} \text { for } i=2,3, \ldots, M-1
$$

Finally at $y=y_{0}$ we have, $\psi_{i, N}=(i-1) h$ and $\psi_{i, N+1}=\psi_{i, N-1}$ for $i=2,3, \ldots, M-1$

The scheme in (13) can be solved uniquely by introducing fictitious nodes externally through central difference formula for the derivative conditions on the boundary. Now by eliminating known boundary values, the equation (13) can be written in the form:

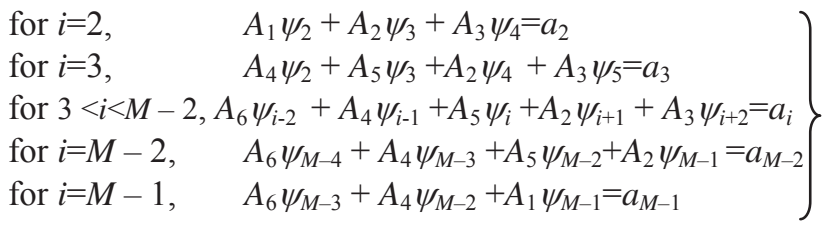

These equations in (18) are solved by Gauss-siedal iteration method. All $\psi_{i}, i>2$ are set to zero and equation for $i=2$ is solved for $\psi_{2}$, then equation for $i=3$ is solved for $\psi_{3}$. So on to find all $\psi_{i}$. For the next iteration, all these are taken as known and the procedure is repeated until, the difference between two successive iterations for $\psi$ is less than $\varepsilon\left(=10^{-4}\right)$.

\subsection{Temperature}

The energy equation, given by (6) can be reduced to:

$$
\begin{gathered}
\nabla^{2} \theta=P e\left[\frac{\partial \psi}{\partial y} \frac{\partial \theta}{\partial x}-\frac{\partial \psi}{\partial x} \frac{\partial \theta}{\partial y}\right]-\frac{H a^{2} \cdot E c \cdot P r}{\left(1+m^{2}\right)}\left[\left(\frac{\partial \psi}{\partial y}\right)^{2}+\left(\frac{\partial \psi}{\partial x}\right)^{2}\right] \\
+\operatorname{Pr} \cdot E c\left[\left(\frac{\partial^{2} \psi}{\partial x \partial y}\right)^{2}+\left(\frac{\partial^{2} \psi}{\partial y \partial x}\right)^{2}\right]
\end{gathered}
$$

with boundary conditions:

$$
\begin{gathered}
\theta=0 \text { on } x=0, \theta=1 \text { on } y=0, \\
\frac{\partial \theta}{\partial x}=q_{1} \text { on } x=1 \text { and } \frac{\partial \theta}{\partial y}=q_{2} \text { on } y=y_{0}
\end{gathered}
$$

$$
-\left[4+\operatorname{Pe}\left(\psi_{i, j+1}-\psi_{i, j-1}\right)\right] \theta_{i-1, j}-\left[4-P e\left(\psi_{i+1, j}-\psi_{i-1, j}\right)\right] \theta_{i, j-1}
$$$$
+16 \theta_{i, j}-\left[4+\operatorname{Pe}\left(\psi_{i+1, j}-\psi_{i-1, j}\right)\right] \theta_{i, j+1}
$$$$
-\left[4-P e\left(\psi_{i, j+1}-\psi_{i, j-1}\right)\right] \theta_{i+1, j}
$$

$$
\begin{gathered}
=\frac{H a^{2} \cdot E c \cdot P r}{\left(1+m^{2}\right)}\left[\left(\psi_{i+1, j}-\psi_{i-1, j}\right)^{2}+\left(\psi_{i, j+1}-\psi_{i, j-1}\right)^{2}\right] \\
+\frac{P r \cdot E c}{2 h^{2}}\left(\psi_{i+1, j+1}-\psi_{i+1, j-1}-\psi_{i-1, j+1}+\psi_{i-1, j-1}\right)^{2} \\
\quad \text { for } i=2,3, \ldots, M \text { and } j=2,3, \ldots, N .
\end{gathered}
$$

The boundary conditions in (20) are now expressed as: at $x=0, \theta=0$ which implies that

$$
\theta_{1, j}=0 \text { for } j=2,3, \ldots, N
$$

at $y=0, \theta=1$ which implies that

$$
\theta_{i, 1}=1 \text { for } i=2,3, \ldots, M
$$

at $x=1, \frac{\partial \theta}{\partial x}=q_{1}$ which implies that

$$
\theta_{M+1, j}=\theta_{M-1, j}+2 h q_{1} \text { for } j=2,3, \ldots, N
$$

at $y=y_{0}, \frac{\partial \theta}{\partial y}=q_{2}$ which implies that

$$
\theta_{i, N+1}=\theta_{i, N-1}+2 h q_{2} \text { for } i=2,3, \ldots, M
$$

The equation (21) can be put in matrix form:

$$
\left.\begin{array}{ll}
\text { for } i=2: & B_{1} \theta_{2}+B_{2} \theta_{3}=b_{2} \\
\text { for } 2<i<M-2: & B_{3} \theta_{i-1}+B_{4} \theta_{i}+B_{2} \theta_{i+1}=b_{i} \\
\text { for } i=M: & -8 I \theta_{M-1}+B_{5} \theta_{M}=b_{M}
\end{array}\right\}
$$


The equations in (26) for temperature are solved by Gauss-siedal iteration method as in (18) for stream function.

\subsection{Current density}

The non-dimensional current density is given by

$$
\begin{gathered}
\boldsymbol{I}^{*}=J_{1}^{*} \boldsymbol{i}+J_{2}^{*} \boldsymbol{j}, \\
\text { where } I_{1}^{*}=\frac{m^{2} U+m V}{\beta\left(1+m^{2}\right)} \text { and } I_{2}^{*}=\frac{m^{2} V-m U}{\beta\left(1+m^{2}\right)}
\end{gathered}
$$

Eq. (27) can be made dimensionless by introducing the following dimensionless variables:

$$
I_{1}^{*}=J_{1} \frac{V_{1}}{\beta}, J_{2}^{*}=J_{2} \frac{V_{1}}{\beta}, U=V_{1} u \text { and } V=V_{1} v
$$

In terms of these dimensionless quantities, Eq. (27) may be written as

$$
J_{1}=\frac{m^{2} u+m v}{1+m^{2}} \text { and } J_{2}=\frac{m^{2} v-m u}{1+m^{2}} .
$$

Using (10) in (28), we get

$$
\begin{array}{r}
J_{1_{i, j}}=\frac{m^{2}\left(\psi_{i, j+1}-\psi_{i, j-1}\right)+m\left(\psi_{i-1, j}-\psi_{i+1, j}\right)}{2 h\left(1+m^{2}\right)} \\
\text { for } i=1,2, \ldots, M \text { and } j=1,2 \ldots, N \\
J_{2_{i, j}}=\frac{m^{2}\left(\psi_{i-1, j}-\psi_{i+1, j}\right)-m\left(\psi_{i, j+1}-\psi_{i, j-1}\right)}{2 h\left(1+m^{2}\right)}
\end{array}
$$

for $i=1,2, \ldots, M$ and $j=1,2 \ldots, N$

Eqs. (29) \& (30) are the current density in $x$ and $y$ directions respectively.

Current density in the chamber is given by

$$
\begin{aligned}
& \left|J_{i, j}\right|=\sqrt{J_{1_{i, j}}^{2}+J_{2_{i, j}}^{2}} \\
& \quad \text { for } i=1,2, \ldots, M \text { and } j=1,2 \ldots, N
\end{aligned}
$$

\section{Results and Discussion}

For the fluid in the chamber, stream function is solved. Using the stream function, temperature distribution and current density are obtained.

\subsection{Streamlines}

The stream lines generated due to suction at the top wall and injection at the right wall are shown in figures $2 \& 3$. In Fig. 2 \& 3, the effect of Reynolds number and suction parameter are shown respectively. We observed that from Fig. 2, Reynolds number does not effecting fluid flow in the chamber. From Fig. 3, we observed that as suction parameter increases, stream function values also increases.

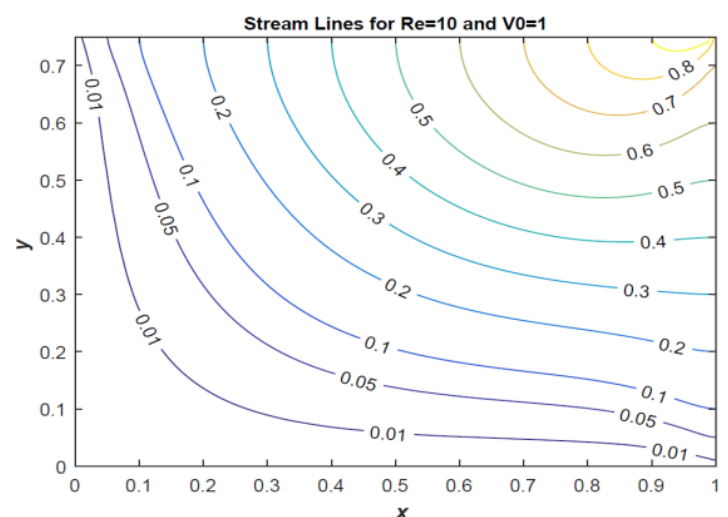

(a)

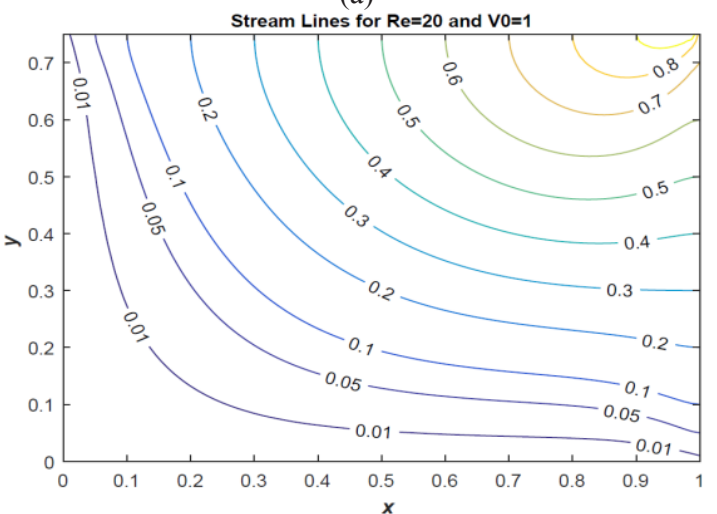

(b)

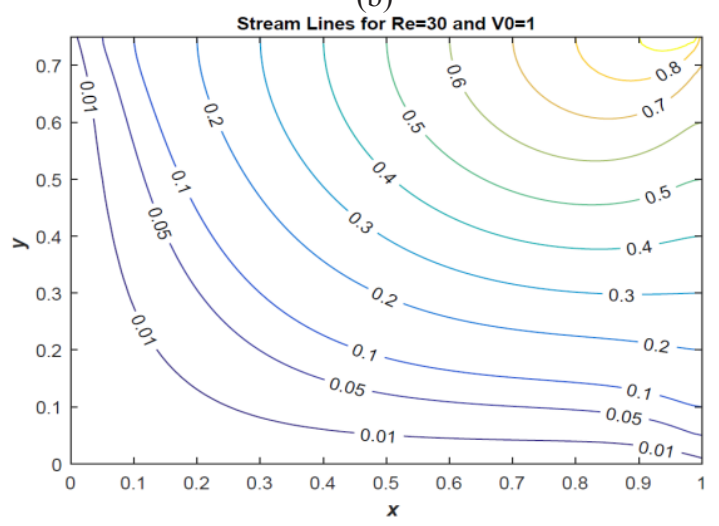

(c)

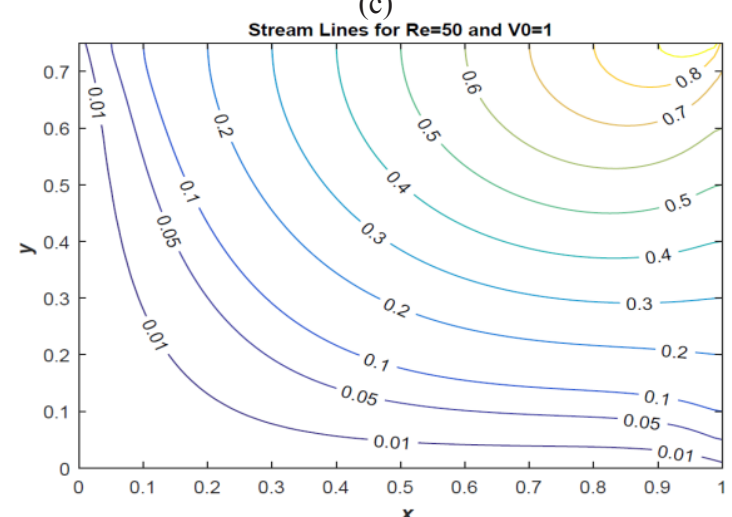

(d)

Fig. 2: Effect of Reynolds number on stream lines at $V_{0}=1$. 


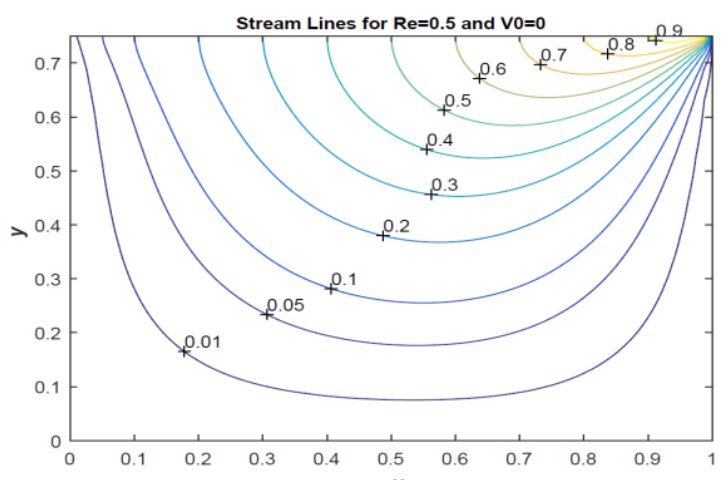

(a) ${ }^{x}$

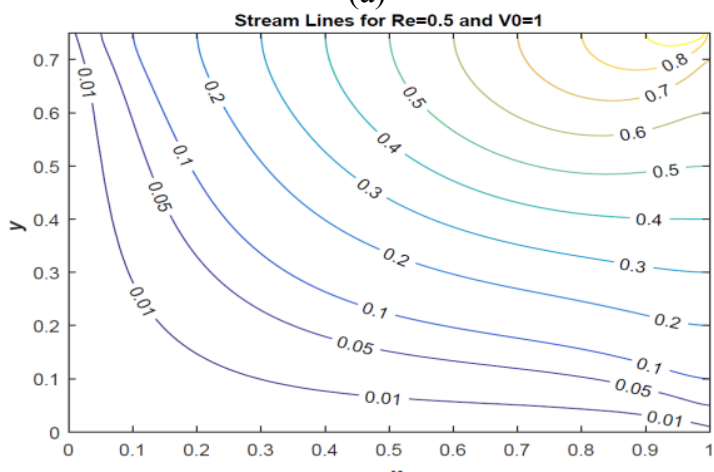

(b)

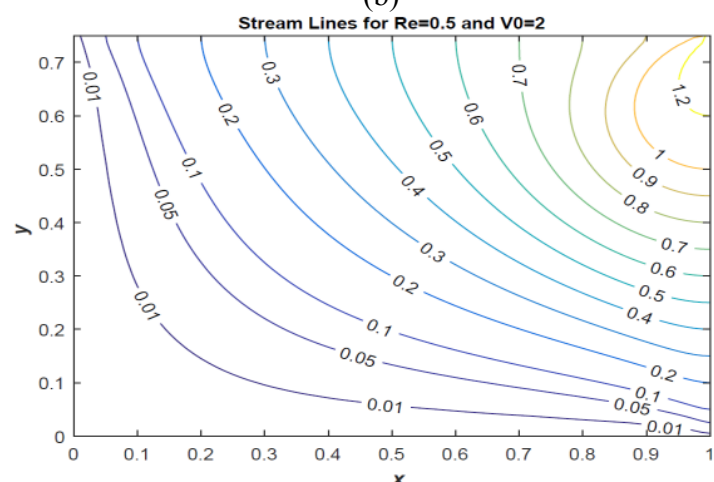

(c)

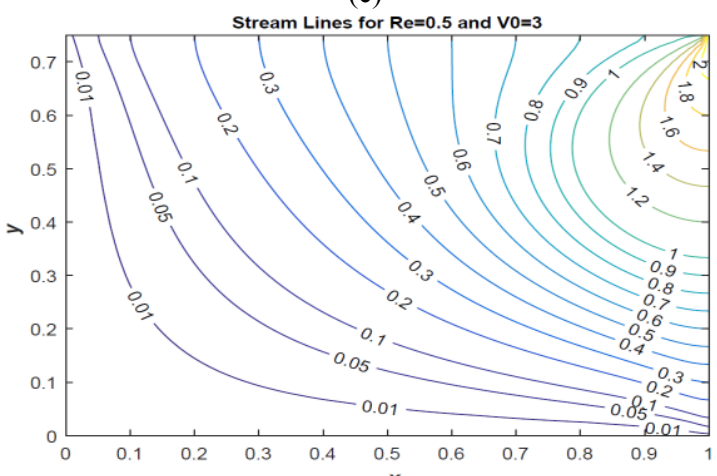

(d)

Fig. 3: Effect of suction parameter on stream lines at $R e=0.5$.

\subsection{Temperature field}

In figures 4, 5 and 6, the effect of Reynolds number, Hartmann number and suction parameter are shown respectively. As the values of Reynolds number $R e$ is increasing, temperature is increasing. As the values of Hartmann number increasing, temperature is also increasing because $\theta$ is proportional to the Hartmann number $\mathrm{Ha}$. As the suction parameter increases, temperature decreases until $V_{0}$ is 1 and when $V_{0}>1$ then increases more and more. We note that the temperature region is divided into two parts: region with $\theta<1$ and region $\theta>1$. When $\operatorname{Re}$ or $H a$ increases, the region $\theta<1$ becomes small.

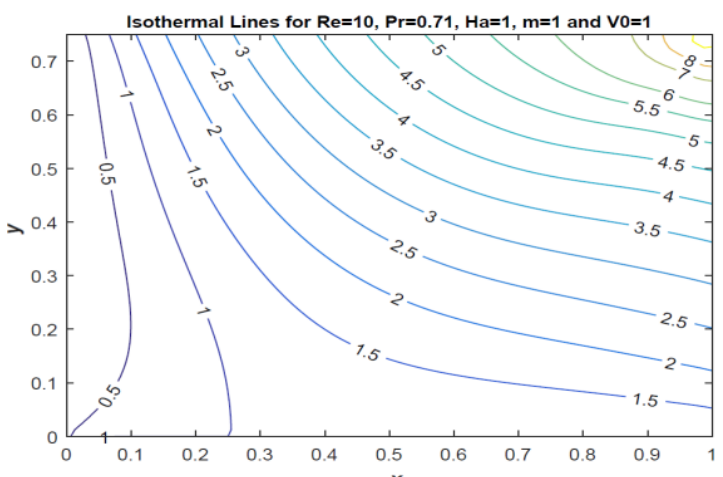

(a)

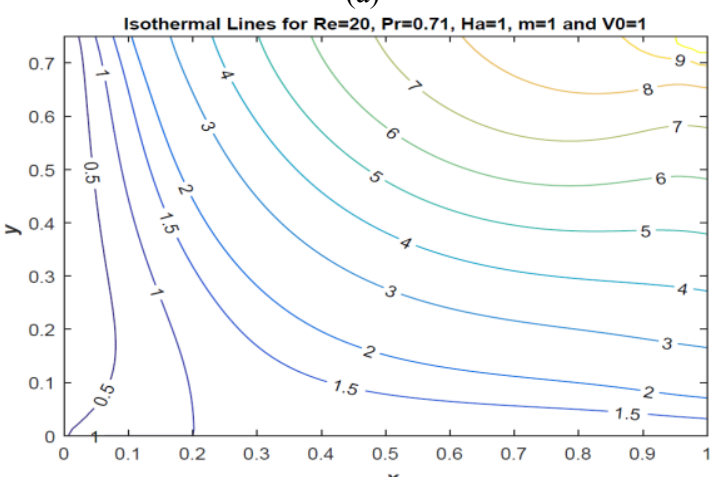

(b)

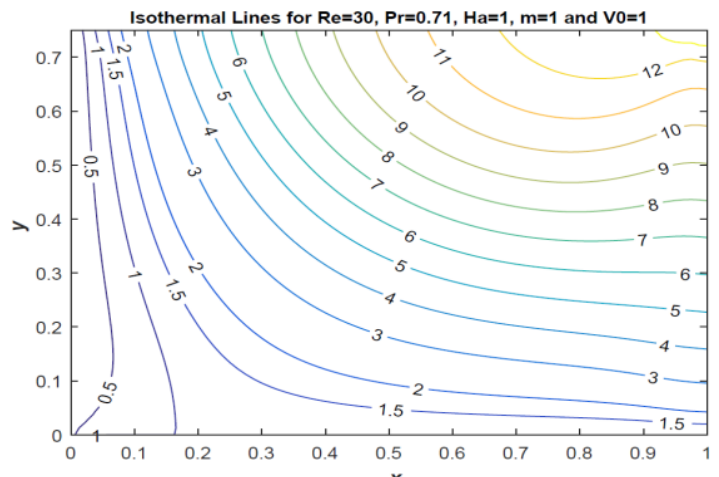

(c)

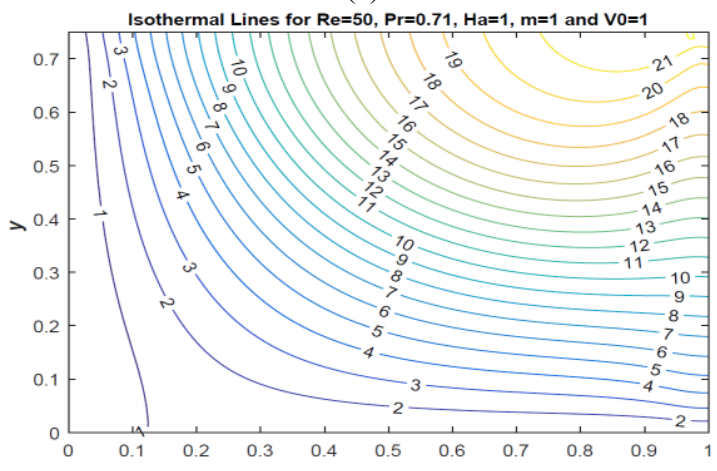

(d)

Fig. 4: Effect of Reynolds number on isothermal lines at $\operatorname{Pr}=0.71, H a=1, m=1$ and $V_{0}=1$. 


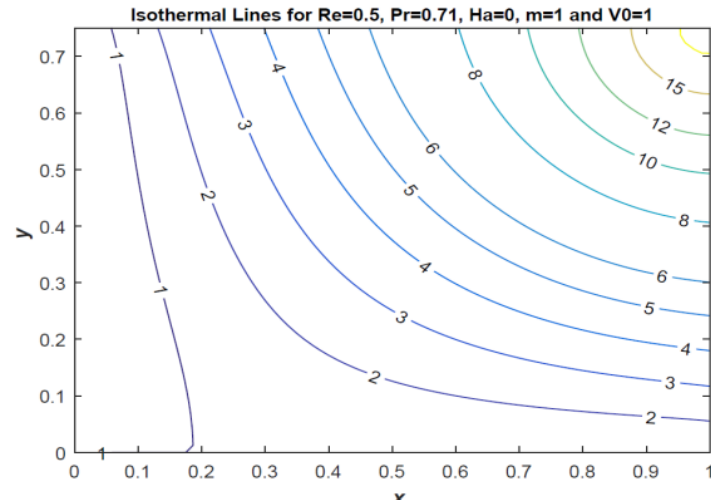

(a)

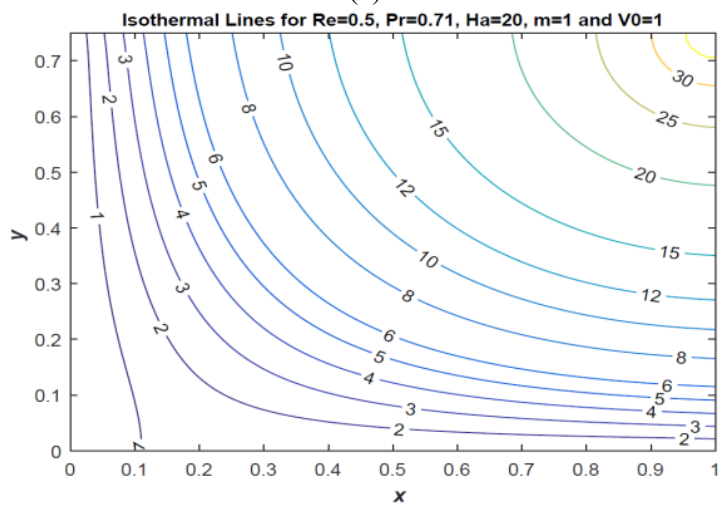

(b)

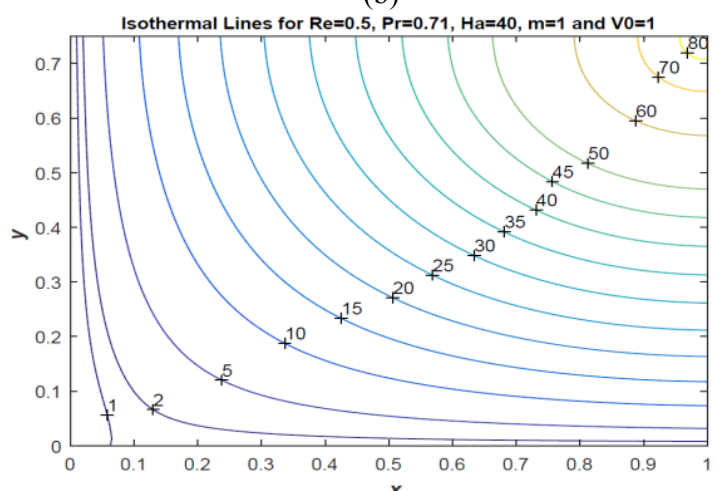

(c)

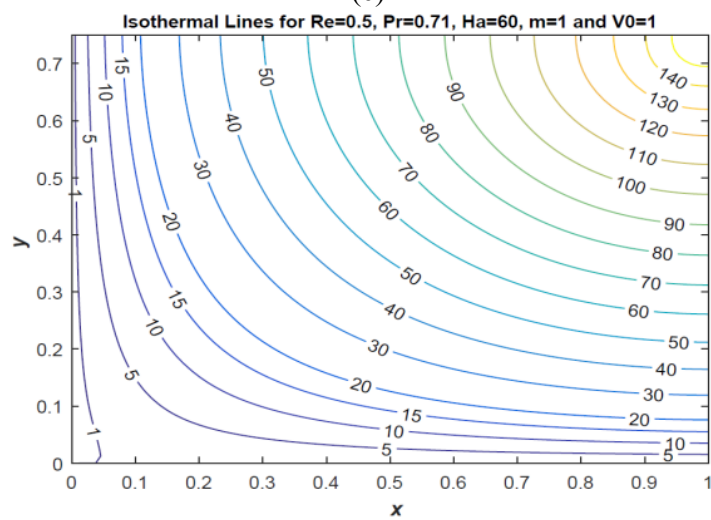

(d)

Fig. 5: Effect of Hartmann number on isothermal lines at $R e=0.5, P r=0.71, m=1$ and $V_{0}=1$.

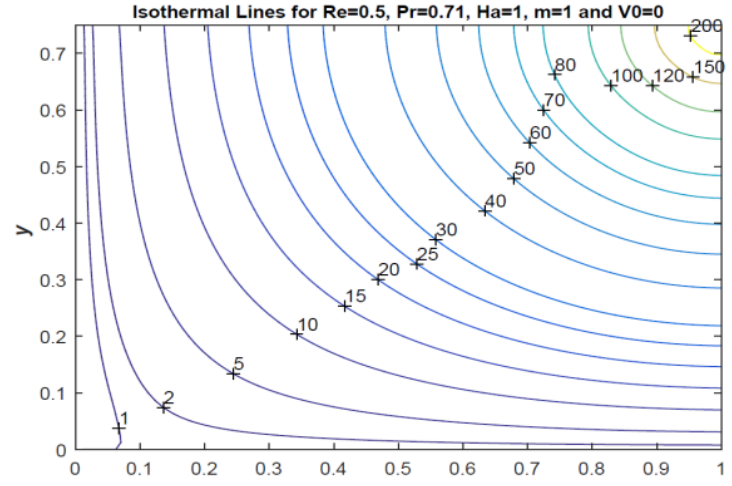

(a)

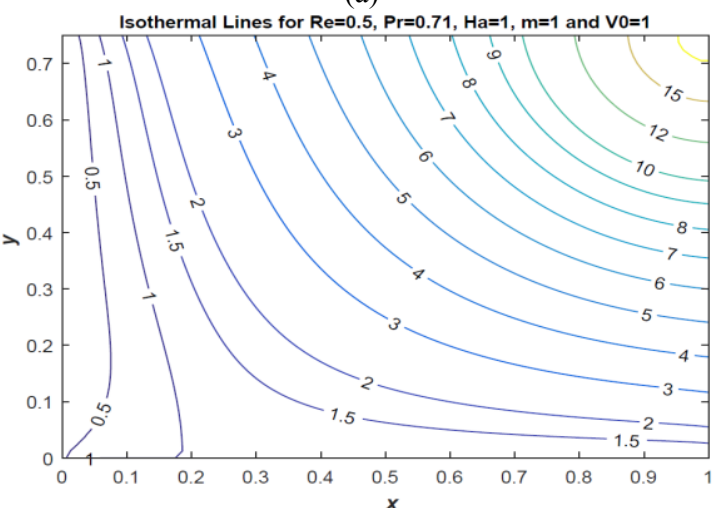

(b)

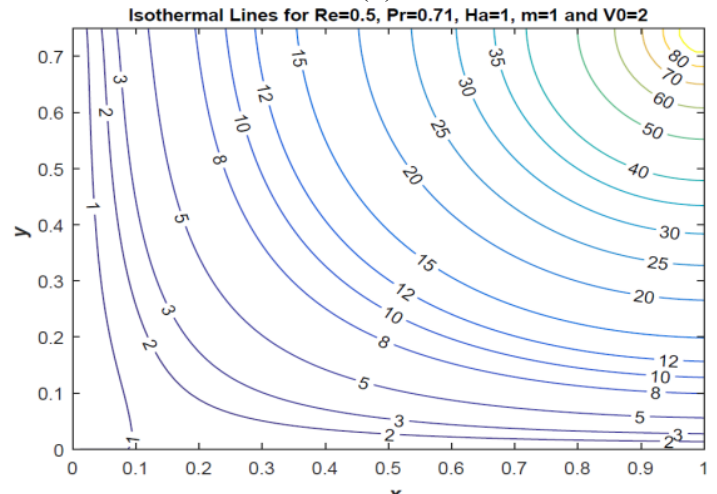

(c)

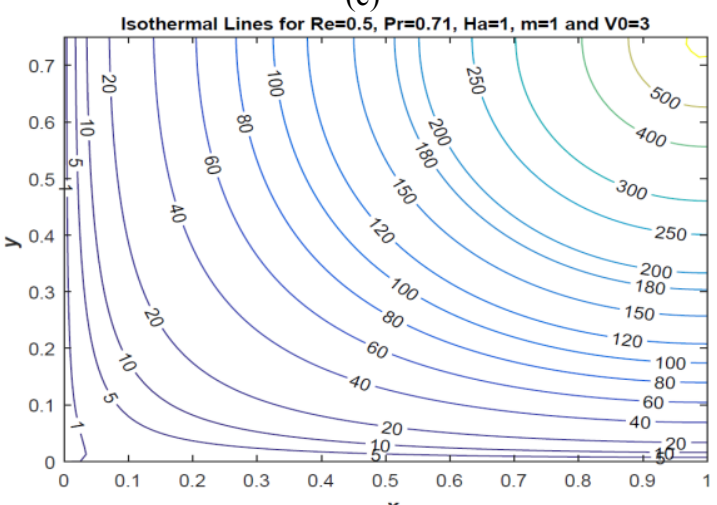

(d)

Fig. 6: Effect of suction parameter on isothermal lines at $R e=0.5, P r=0.71, H a=1$ and $m=1$.

\subsection{Current density}

In figures 7, 8, 9 and 10, the effect of Reynolds number, Hartmann number, Hall parameter and suction parameter 
are shown respectively. When $R e$ increases, the circulation region at the top left corner is divided two circulation regions one at the left top and one at the left bottom. A similar observation can be observed when suction parameter increases.

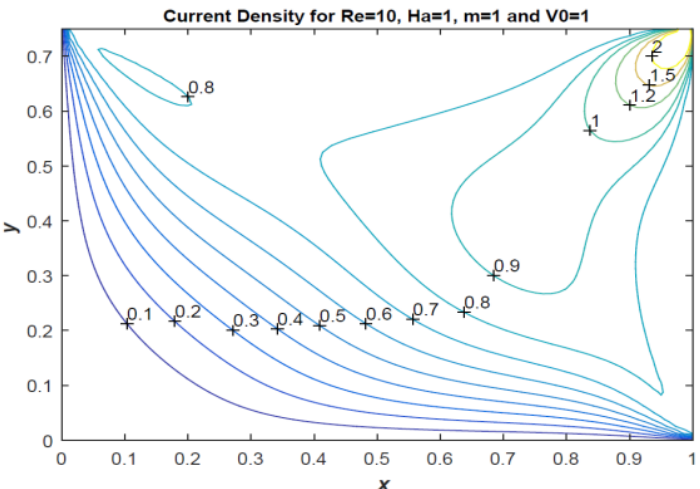

(a)

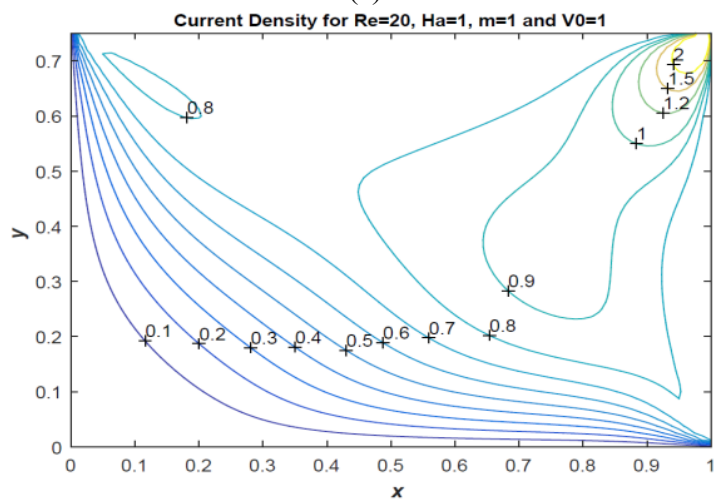

(b)

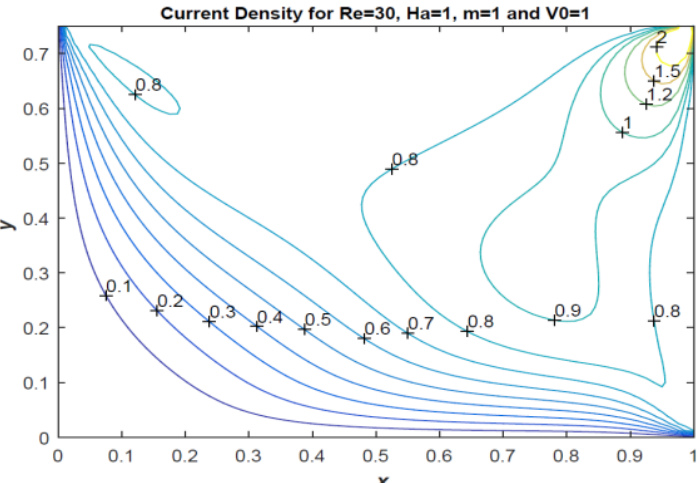

(c)

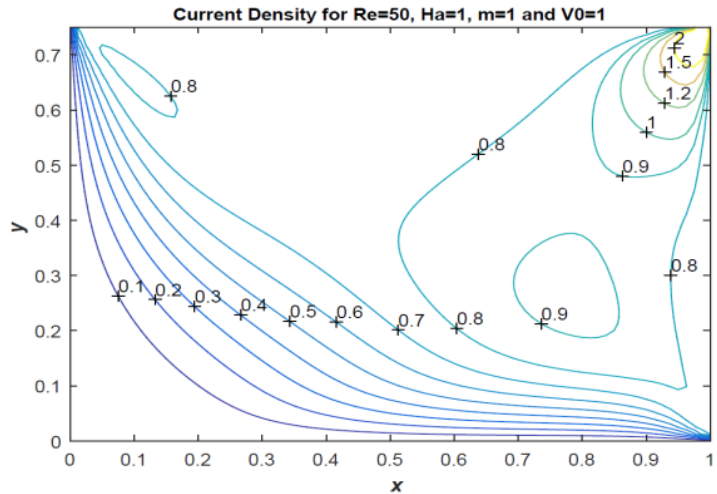

(d)

Fig. 7: Effect of Reynolds number on current density at $H a=1$, $m=1$ and $V_{0}=1$.

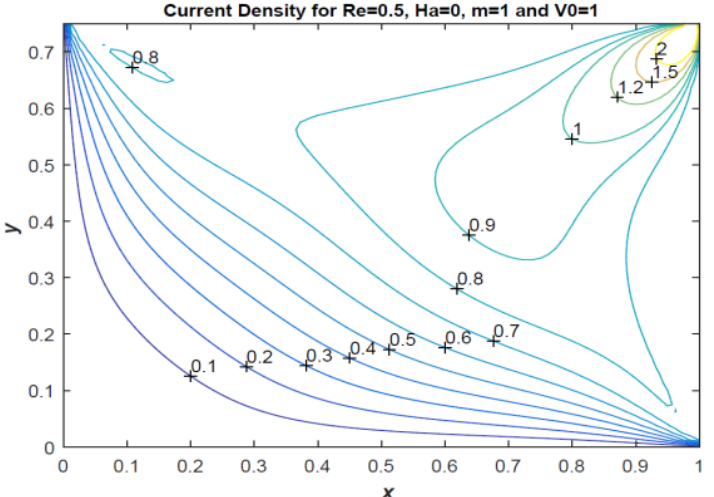

(a)

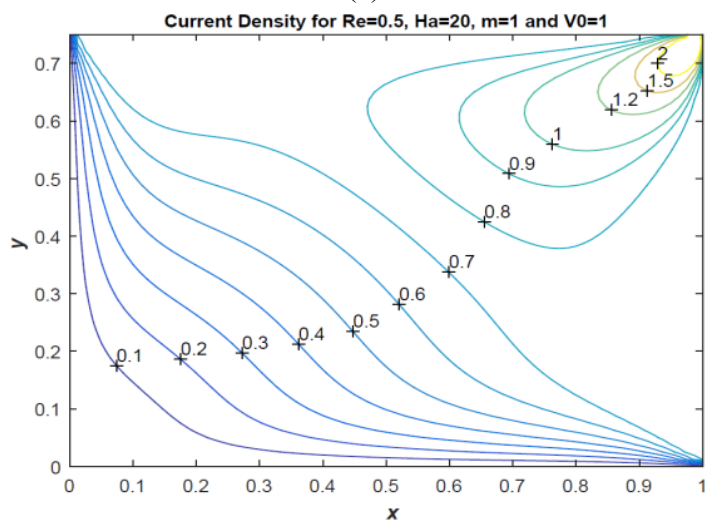

(b)
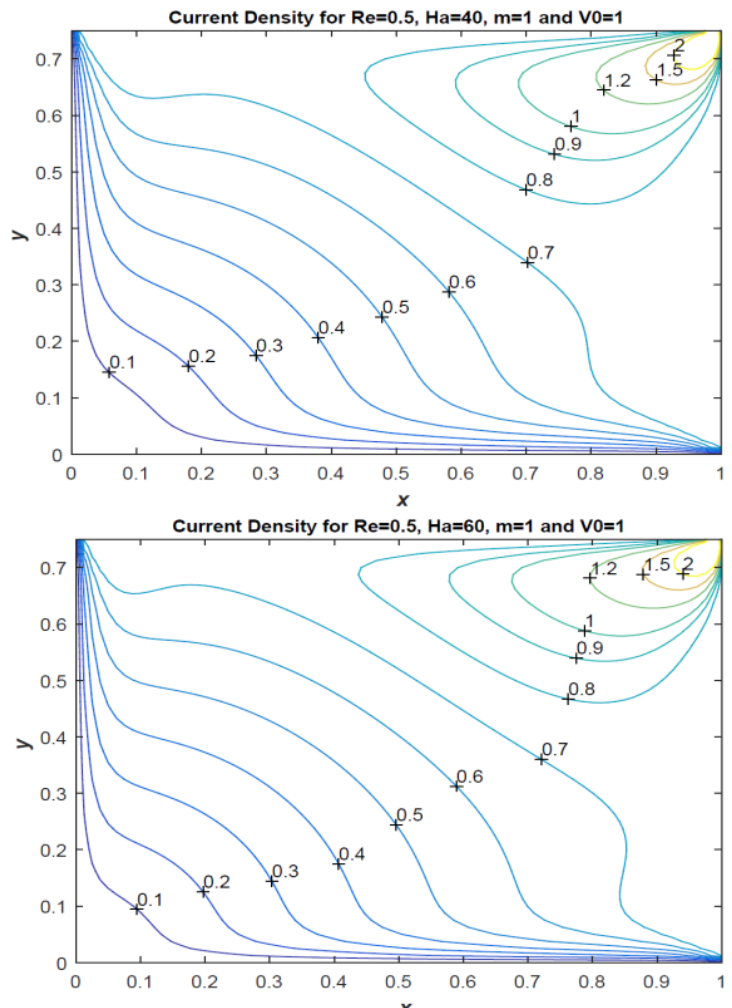

(d)

Fig. 8: Effect of Hartmann number on current density at $R e=0.5, m=1$ and $V_{0}=1$. 


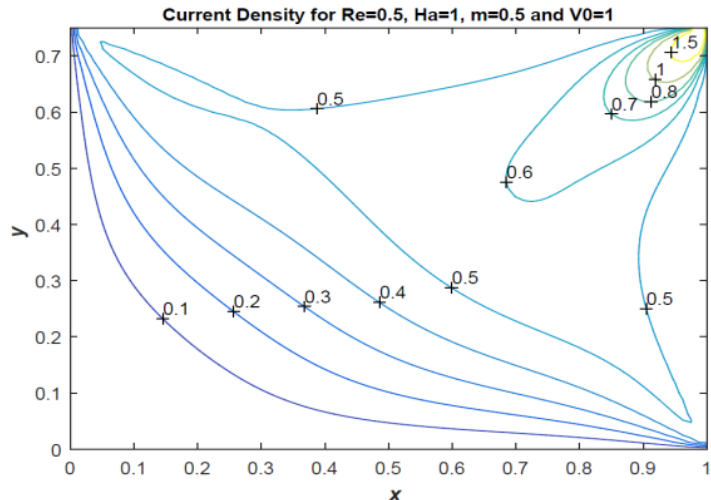

(a)

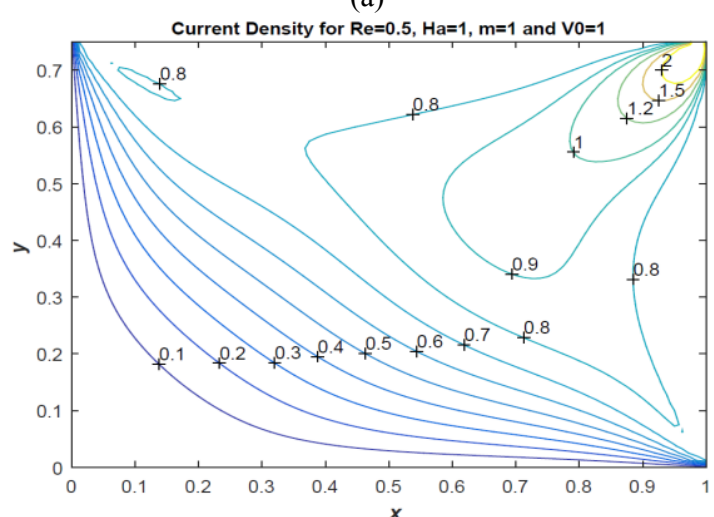

(b)

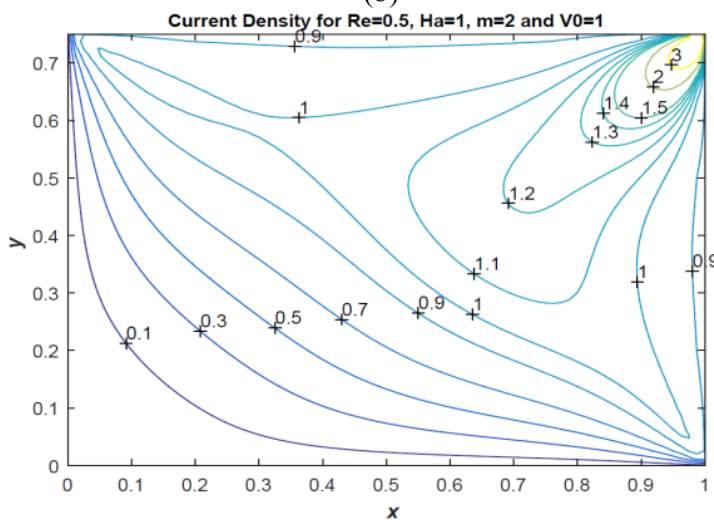

(c)

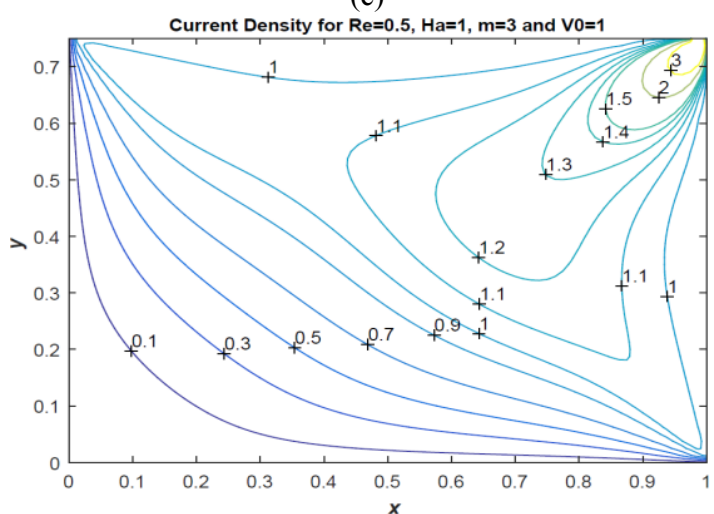

(d)

Fig. 9: Effect of Hall parameter $m$ on current density at $R e=0.5$, $H a=1$ and $V_{0}=1$.

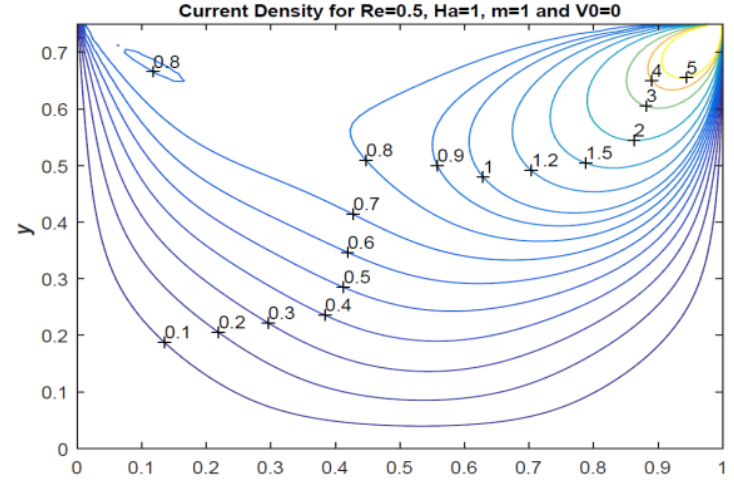

(a)

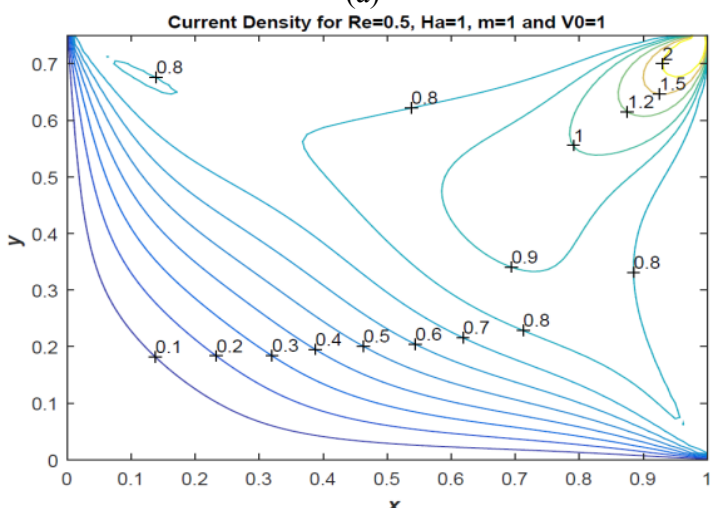

(b)

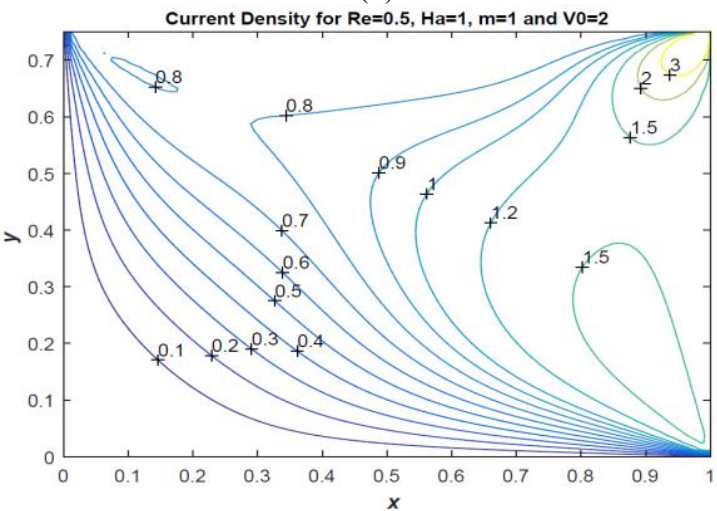

(c)

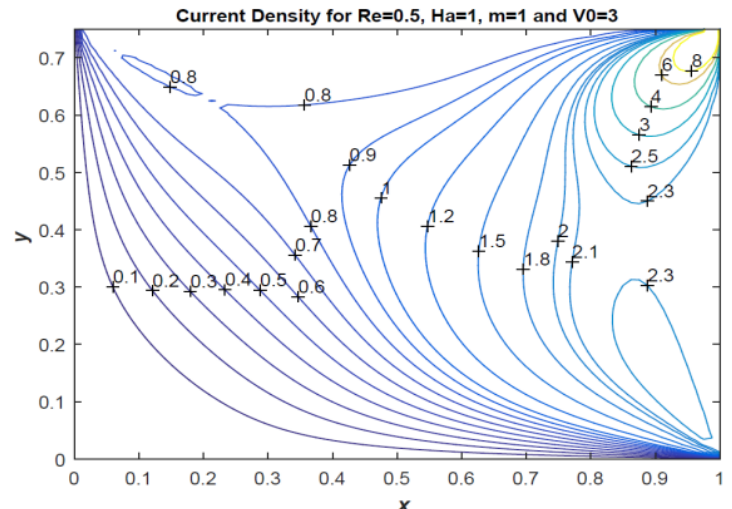

(d)

Fig. 10: Effect of suction parameter on current density at $R e=0.5, H a=1$ and $m=1$. 


\section{Conclusions}

From the above observations we conclude that

$i$. When suction increases, flow into the chamber (values of stream lines) increases.

ii. The temperature in the cavity is divided into region with $\theta<1$ and region with $\theta>1$. The region with $\theta<1$ decreases as Reynolds number or Hartmann number increases.

iii. Current density divides in two circulation regions at the top and bottom of left side when suction or Hall parameter increases.

\section{References}

1. A.S. Berman, Journal of Applied Physics, 24, 1232 (1953).

2. A.S. Berman, Journal of Applied Physics, 29, 71 (1958).

3. J. Hartmann, Hg-Dynamics I. Theory of the Laminar Flow of an Electrically Conductive Liquid in a Homogeneous Magnetic Field, (Kgl. Danske Videnskabernes Selskab, bd. 15, nr. 6, Mathematisk-fysiske Meddelelser, Copenhagen, 1937).

4. J. Hartmann, F. Lazarus, Hg-Dynamics II. Experimental Investigations on the Flow of Mercury in a Homogeneous Magnetic Field, (Kgl. Danske Videnskabernes Selskab, bd. 15, nr. 7, Mathematisk-fysiske Meddelelser, Copenhagen, 1937).

5. J.C.R. Hunt, Journal of Fluid Mechanics, 21, 577 (1965).

6. K.N. Mehta, R.K. Jain, 28, A, 846 (1961).

7. T.S. Lundgren, B.H. Atabek, C.C. Chang, The Physics of Fluids (1958-1988), 4, 1006 (1961).

8. K.K. Mandal, Applied Science Research., 19, 97 (1968).

9. J.V. Ramana Murthy, K.S. Sai, N.K. Bahali, AIP Advances, 1 (2011).

10. K.S. Sai, B. Nageswara Rao, Acta Mechanica, 140, 57 (2000).

11. V.M. Soundalgekar, N.V. Vighnesam, H.S. Takhar, IEEE Transactions on Plasma Science, PS-7, 178 (1979).

12. V.M. Soundalgekar, A.G. Uplekar, IEEE Transactions on Plasma Science, PS-14, 579 (1986).

13. Itiro Tani, J. of the Aerospace Sci, 297 (1962).

14. M.E.S. Ahmed, H.A. Attia, Int. Comm. Heat and Mass Transfer, 27, 1177 (2000).

15. M.E.S. Ahmed, H.A. Attia, Canadian Journal of Physics, 83, 637 (2005).

16. A.L. Aboul-Hassan, H.A. Attia, Canadian J. of Physics, 80, 579 (2002).

17. D. Srinivasacharya, Mekonnen Shiferaw, J. of the Braz. Soc. of Mech. Sci. \& Eng., 4, 313 (2008).

18. H.A. Attia, M.E.S. Ahmed, Applied Mathematical Modeling, 28, 1027 (2004).

19. H.A. Attia, Turkish Journal of Engineering and Environmental Sciences, 30, 285 (2006).
20. Titus Petrila, Damian Trif, Basics of Fluid Mechanics and Introduction to Computational Fluid Dynamics (Springer Science + Business Media, Boston 2005).

21. C. Pozrikidis, Numerical Computation in Science and Engineering (Oxford University Press, Oxford, 1998). 